EOMmUn: Communication et organisation

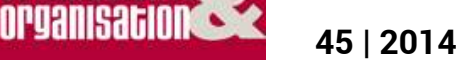

Risques mineurs, changements majeurs

\title{
Communiquer, prévenir, éduquer
}

Circulation de normes et de savoirs sur les risques alimentaires à l'école Communicating, preventing, educating: the circulation of norms and knowledge on the dietary risks in school

\section{Simona De lulio et Susan Kovacs}

\section{OpenEdition \\ Journals}

Édition électronique

URL : http://journals.openedition.org/communicationorganisation/4512

DOI : 10.4000/communicationorganisation.4512

ISSN : $1775-3546$

Éditeur

Presses universitaires de Bordeaux

\section{Édition imprimée}

Date de publication : 1 juin 2014

Pagination : $99-114$

ISBN : 978-2-86781-904-9

ISSN : $1168-5549$

Référence électronique

Simona De lulio et Susan Kovacs, "Communiquer, prévenir, éduquer », Communication et organisation [En ligne], 45 | 2014, mis en ligne le 01 juin 2017, consulté le 01 mai 2019. URL : http:// journals.openedition.org/communicationorganisation/4512; DOI : 10.4000/ communicationorganisation.4512 


\title{
Communiquer, prévenir, éduquer circulation de normes et de savoirs sur les risques alimentaires à l'école
}

\author{
Simona De lulio' et Susan Kovacs²
}

\section{Introduction}

Parmi les différentes inquiétudes qui traversent la vie des sociétés occidentales (Lagadec, 1981; Giddens, 1984 ; Beck, 2001), les risques associés à l'enfance et à l'alimentation sont aujourd'hui au centre de l'arène publique. Depuis très longtemps appréhendés comme source de périls et de peurs, les enfants et leurs conduites alimentaires sont associés à des risques multiples qui catalysent l'attention des milieux médicaux, sociaux, éducatifs, politiques et affectent les pratiques d'un ensemble très hétérogène d'acteurs (familles, écoles, entreprises du secteur agro-alimentaire, institutions gouvernementales, collectivités territoriales, médias).

Comme la sociologie et l'anthropologie du risque l'ont montré, les objets, les actions, les situations envisagés comme dangereux varient selon les cadres socio-culturels et historiques. Les « objets de risque " (Hilgartner, 1999 ; Lupton, 1999) ne constituent pas des entités statiques, mais des catégories continuellement façonnées et négociées en tant que parties d'un réseau d'interactions socio-culturelles. Selon cette perspective, la réalité sociale des risques se fabrique à travers des processus discursifs, des débats et des controverses qui participent à l'identification des périls, des responsabilités et des sources de danger. Dans ce cadre, les dispositifs d'information et de communication jouent un rôle décisif non seulement dans la perception, mais aussi dans la configuration, la circulation et la « trivialisation » (Jeanneret, 2008) des catégories de risques. En l'occurrence, ils constituent des sites d'observation particulièrement appropriés pour étudier les différentes logiques d'action autour des risques liés à l'alimentation des enfants (De Iulio, 2011).

1 Simona De Iulio est professeure en Sciences de l'information et de la communication à l'Université de Lille 3. Elle est membre du Groupe d'Études et de Recherche Interdisciplinaire en Information et Communication (GERiiCO) ; simona.deiulio@univ-lille3.fr

2 Susan Kovacs est maître de conférences en Sciences de l'information et de la communication à l'Université de Lille 3. Elle est membre du Groupe d'Études et de Recherche Interdisciplinaire en Information et Communication (GERiiCO) ; susan.kovacs@univ-lille3.fr 
L'objectif de cet article est d'examiner la rhétorique des risques alimentaires présente dans les dispositifs d'information et de communication produits par les organisations qui travaillent dans le secteur de la restauration scolaire. Quel type de savoirs, de normes et de comportements liés aux risques alimentaires sont performés par ces dispositifs ? Quelle place occupent-ils en relation aux dispositifs pédagogiques traditionnels (polycopiés, manuels) et ouvrages de vulgarisation ciblant les jeunes, sur les questions de nutrition et d'alimentation ? Nous essayerons de donner une réponse à ces questions à partir de l'analyse des résultats rassemblés dans le cadre du projet de recherche interdisciplinaire "L'alimentation scolaire : acteurs, pratiques et discours en Nord - Pas de Calais » réalisé avec le soutien de Lille Métropole Communauté Urbaine et de la Fondation Louis Bonduelle. Ce projet a pour objectif d'analyser les logiques des acteurs (institutions, entreprises, éducateurs, parents, élèves) à travers les discours et les pratiques produits au sein de la communauté éducative qui participent à la construction de savoirs, valeurs, normes et opinions sur l'alimentation et la nutrition des élèves, dans et autour de la cantine scolaire.

Dans cet article, nous proposons une analyse sémio-pragmatique d'un corpus constitué d'un côté de dispositifs info-communicationnels produits par des entreprises françaises de restauration collective et de l'autre côté, de dispositifs élaborés par et pour les acteurs éducatifs et les élèves dans le cadre des programmes ministériels sur l'alimentation et des préconisations du Ministère de l'Éducation pour la mise en place d'une éducation à la santé et d'une éducation nutritionnelle (Manderscheid, Pithon, 2000). Plus précisément, notre corpus est composé des sites web d'Api Restauration, Elior, Scolarest, Sodexo ainsi que des kits pédagogiques, newsletters, affiches qui sont téléchargeables à partir de ces sites et qui s'adressent aux élèves, aux parents, au personnel enseignant et non enseignant des établissements scolaires tout comme aux collectivités territoriales. Lintérêt pour ces dispositifs est lié au constat que, au cours des deux dernières décennies, les entreprises de restauration scolaire ont commencé à valoriser leurs prestations à travers des initiatives info-pédagogiques en participant ainsi aux discours éducatifs autour de l'alimentation au sein des écoles (Dubuisson-Quellier, 1999). Nous nous sommes ainsi intéressées aux dispositifs info-communicationnels réalisés par ces organisations à des fins à la fois éducatives ou de sensibilisation et de communication.

Afin de dégager les spécificités de ces dispositifs, nous les avons comparés à trois types de documents provenant de sources proches du monde de l'école : des polycopiés choisis ou adaptés par des enseignants à partir de manuels ou sites web de partage de ressources pédagogiques, un livret pédagogique produit par l'INPES à destination des élèves de l'école primaire (Léo et la terre), et un ensemble d'ouvrages documentaires de vulgarisation sur la nutrition et l'alimentation produits par des maisons d'édition connues par le monde 
éducatif (Actes Sud Junior, Casterman, Gallimard Jeunesse, Magnard). Les polycopiés analysés sont ceux utilisés par des enseignants de deux écoles primaires de la région lilloise dans les séquences consacrées à l'alimentation ; les ouvrages documentaires étudiés ont été choisis 1) parmi une sélection faite par des enseignants de la ville de Lille et gardée à leur attention dans une réserve à la médiathèque municipale, et 2) parmi des ouvrages figurant dans un kit pédagogique préparé par la Maison régionale de l'environnement et des solidarités de Lille ${ }^{3}$.

Nous allons nous intéresser, tout d'abord, aux causes de danger identifiées dans ces dispositifs : quelles substances ou actions sont susceptibles de provoquer des effets néfastes ? Comment sont-elles présentées sur le plan linguistique et visuel ? Avec quels attributs ? Nous allons ensuite interroger les façons dont les dangers sont caractérisés et examiner quels types de dommages sont mis en avant dans ces dispositifs. Communique-t-on sur la probabilité que ces conséquences se produisent, c'est-à-dire sur le « niveau de risque »? Communique-t-on sur la temporalité et la gravité des effets (immédiats ou à long terme, dommages vitaux ou chroniques ... ) ? Nous allons, enfin, nous focaliser sur les modalités de maîtrise des risques alimentaires, sur les remèdes proposés, l'identification des acteurs censés prendre des mesures pour éviter ou réduire le risque ainsi que sur les stratégies de responsabilisation mises en place. En tentant de donner des réponses à tous ces questionnements, nous essayerons également de rendre compte des modalités de légitimation des savoirs sur les risques alimentaires et de construction de la confiance. Qu'estce qui confère crédibilité à ces discours ? Quelle est la place des savoirs savants, experts, spécialisés, profanes et d'expérience dans la rhétorique des risques alimentaires ? Quel rôle est joué par les croyances largement partagées?

Nous observons la scénarisation des risques liés à l'alimentation dans des dispositifs produits par des acteurs divers (entreprises de restauration scolaire, éditeurs et auteurs relevant du secteur de l'éducation ou de la vulgarisation scientifique pour jeunes) afin de repérer les invariants et les spécificités discursives dans la construction d'un rapport entre les élèves et les risques alimentaires. En quoi les discours des entreprises sont-ils comparables à ceux produits par d'autres acteurs, et comment peut-on comprendre les rapprochements ou divergences, par rapport aux positionnements ou stratégies communicationnelles des concepteurs de ces supports ? Dans les deux cas, le discours sur le risque "entre » dans l'école par un biais non-traditionnel. La légitimité de l'entreprise, comme celle d'une " éducation à la santé » censée mobiliser les équipes éducatives autour de projets de sensibilisation et d'action concertée, ne va pas de soi au sein de l'école (Jourdan, et al., 2002). Nous verrons donc à quel point les stratégies discursives mises en place par

3 Pour une description de ce kit voir http://mres-asso.org/spip.php?breve333. 
ces acteurs pour construire la notion de risque relèvent d'un discours normé, à la fois culpabilisant et rassurant, où les dangers apparaissent à la fois réels et insaisissables.

\section{Les causes de danger entre l'implicite, la caricature et le non-dit}

Dans la plupart des dispositifs info-pédagogiques et de communication autour de l'alimentation à l'école proposés par les entreprises de restauration collective, les informations concernant les causes de danger sont placées en arrière-fond. Les causes de danger, c'est-à-dire les substances, les aliments et/ ou les actions susceptibles de provoquer des effets néfastes, sont très souvent présupposées et ne sont pas mentionnées de manière ouverte. Les dispositifs info-pédagogiques portant surl'alimentation ne fournissent pas d'informations claires et explicites aidant à identifier avec précision les sources de risques qui demeurent tacitement sous-entendues. "Mal manger ", " manger n'importe quoi, à n'importe quelle heure », « mauvaise alimentation » sont certaines des formules génériques et allusives, tirées du langage quotidien, avec lesquelles les pratiques alimentaires nuisibles sont désignées. Les sources de péril semblent ainsi faire partie des évidences partagées par l'ensemble des destinataires de ces discours.

Rarement des modes et des types de consommation alimentaire jugés nuisibles sont définis de façon précise. La " monotonie », les " grignotages ", le «fast-food », les « snacks », les « barres chocolatées », les " céréales fourrées » les " portions trop abondantes ", « trop de sucres et de graisses ", les " plats riches en lipides » sont sporadiquement indiqués comme des conduites alimentaires, des aliments et des substances dommageables. Dans certains cas, c'est à travers la mise en image caricaturale que les aliments et les conduites à éviter sont affichés. Un cornet de glace géant est, par exemple, associé à l'image d'un garçon potelé qui illustre les initiatives de « lutte contre l'obésité et les déséquilibres alimentaires " de l'entreprise de restauration collective Scolarest. Autre exemple, une des couvertures de la newsletter De bouche à oreille adressée aux parents et aux élèves par cette même entreprise, présente l'image d'une fille avec la bouche grande ouverte en train de mordre dans un énorme hamburger sur lequel en surimpression apparaît le pictogramme de la croix évoquant le symbole qui dans le système européen de signalisation des substances dangereuses indique les substances et préparations toxiques. Si les sources de risque demeurent sous-entendues, citées de façon allusive ou mises en image de façon caricaturale, les raisons qui amènent certaines conduites, aliments et substances à nuire à l'individu et à la collectivité relèvent du nondit. Les dispositifs info-pédagogiques et communicationnels produits par les entreprises de restauration collective omettent d'éclairer la chaîne causale qui relie les objets et les actions jugés nuisibles aux dangers correspondants.

L'ensemble de ces dispositifs apparaît, en effet, traversé par une conception fonctionnaliste de l'acte de manger, largement inspirée de la nutrition 
et de la diététique. Il s'agit d'une conception qui amène à reconnaître les besoins de l'organisme humain, les fonctions de chaque aliment et de chaque substance et à " préciser les principes et la pratique d'une nutrition humaine rationnelle [italique notre]» (Heller et al., 2014). Dans ces dispositifs info-pédagogiques, ce sont surtout les apports nutritionnels des différents aliments qui sont mis en relief alors que les conséquences dommageables de certaines substances, ingrédients, plats, modes de consommation sont passées sous silence. Ces documents n'indiquent pas ouvertement les conduites, les aliments, les substances à éviter et les raisons pour lesquelles il faut les éviter. En revanche, ils suggèrent de façon très explicite ce qu'il faut faire, les conduites à tenir et les aliments à privilégier. En particulier, certains de ces dispositifs entrent en résonance avec les recommandations du Programme National Nutrition Santé (PNNS). La campagne d'animation au sein des restaurants scolaires « Les super héros de l'alimentation » réalisée par Scolarest en janvier 2011, par exemple, relance ouvertement les exhortations du PNNS en les associant à des dessins et à un graphisme propre aux produits culturels de l'enfance (figure $n^{\circ} 1$ ). À l'origine du danger il y aurait donc implicitement le fait de ne pas adhérer aux normes proposées, ne pas évaluer et contrôler les prises alimentaires, ne pas prendre toutes les mesures nécessaires pour prévenir les dangers comme les savoirs experts des nutritionnistes et les savoirs institutionnels le recommandent.

Fig. $n^{\circ} 1$ : Campagne d'animation au sein des restaurants scolaires "Les super-héros de l'alimentation " réalisée par Scolarest en janvier 2011

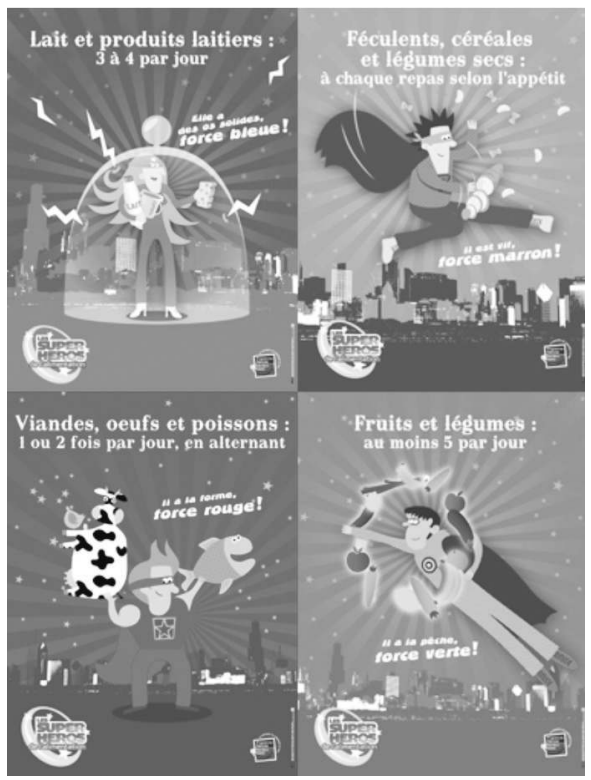

Ces implicites et ce non-dit caractérisent non seulement les dispositifs info-pédagogiques et communicationnels des entreprises de restauration 


\section{$\mathrm{C} \& O \mathrm{n}^{\circ} 45$}

scolaire, mais aussi les documents produits par des acteurs éducatifs ou des éditeurs d'ouvrages de vulgarisation sur la nutrition ciblant un public de jeunes. Ici, le corps est présenté comme une machine (ou une usine) qui a besoin d'aliments pour survivre. Les comportements sont évalués par leur effet sur l'équilibre entre dépense énergétique et ingestion d'aliments en tant que source d'énergie. L'aliment chasse donc le risque (figure $n^{\circ} 2$ ).

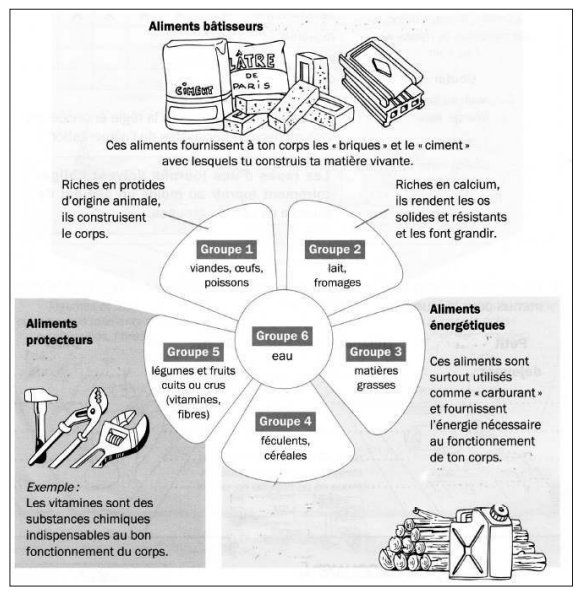

Fig. $n^{\circ} 2$ : un polycopié utilisé dans une classe de CE 2. Les aliments protecteurs éloignent le danger des incommodités de la vie, telles que maladies, froid, allergies

La référence à des causes psychologiques ou héréditaires d'une prise excessive de poids est rare ou ponctuelle et présentée comme un problème spécifique à " certaines personnes ", et qui implicitement, ne concerne pas directement le jeune lecteur/élève, ou alors lui sert de contrexemple. Dans une étude de cas d'un garçon en surpoids, par exemple, au sein de l'ouvrage documentaire de vulgarisation Les Aliments, à petits pas ${ }^{4}$, on apprend que «Lucas » est jaloux de son petit frère et que, par conséquent, il mange trop. Ce contexte familial est pourtant bien vite mis de côté par un narrateur qui explique au lecteur que Lucas a pris « la mauvaise habitude d'avoir toujours quelque chose à grignoter dans ses poches » : la source du problème est ramenée à une conduite à corriger et non à une souffrance psychologique, détail qui semble appartenir à la singularité de la situation de «Lucas ». Le lecteur, lui, appartient implicitement à la catégorie universelle de jeunes qu'il s'agit de convaincre du bien fondé des normes nutritionnelles.

Les documents utilisés dans la classe (cycle 2, école primaire) lors de séquences d'enseignement en lien avec les programmes sur l'alimentation, abordent cette thématique uniquement par le biais du comportement nutritionnel : le risque, s'il apparaît, survient uniquement par rapport aux choix de l'individu. En revanche, dans les documents de vulgarisation pour jeunes, souvent de portée plus large (abordant non seulement la nutrition,

4 Actes Sud Junior, 2008. 
mais aussi l'histoire des pratiques alimentaires, la gastronomie, les cultures culinaires, et l'industrialisation de la production alimentaire) et proposés dans des bibliothèques d'école ou dans des kits pour animateurs ou enseignants, les risques alimentaires liés à des pratiques agricoles ou industrielles se verront abordés explicitement : « comment les nitrates ont-ils pu s'infiltrer dans l'eau ? À cause des engrais répandus dans les champs [...] Ces engrais passent au travers de la terre jusque dans les cours d'eau et les nappes souterraines... » (Les Aliments à petits pas, 2008). Ces risques extérieurs aux conduites individuelles sont développés, notamment par rapport aux causes et à l'enchaînement des phénomènes qui les font parvenir chez l'homme, d'une manière somme tout inquiétante, malgré les tentatives de mettre en place un discours rassurant sur les contrôles sanitaires qui existent aujourd'hui. En effet, si la cause est connue, les conséquences sont graves et les solutions n'existent pas forcément. On constate donc une différence de traitement, dans les documents de vulgarisation, entre le domaine des choix nutritionnels de l'enfant, où le risque est " maîtrisé ", et celui des menaces qui dépassent le contrôle individuel et qui sont représentés selon une logique du discours sensationnel. Tout comme le discours de l'entreprise, le discours didactique (manuels, polys) occulte le plus souvent les dangers, y compris les risques industriels, alors que le discours de vulgarisation les exhibe et les médiatise.

De même, les ouvrages sur les aliments et l'alimentation qui sont d'orientation écologique (tels que ceux qui figurent dans le kit «De mon assiette à ma planète » conçu par la Maison régionale de l'environnement et des solidarités - MRES - de Lille pour les enfants de l'école primaire) dressent souvent un portrait alarmant des techniques industrielles qui, parfois à l'insu de l'individu, sont causes de risques alimentaires. À travers des exemples tels que l'irradiation des boîtes de conserve et la présence des additifs on cherche à faire prendre conscience à l'enfant des pratiques parfois peu éthiques des acteurs de l'industrie agroalimentaire. L'objectif ici est de soulever un sentiment d'injustice chez l'enfant, tout en lui faisant peur. Cependant, le discours nutritionnel qui écarte le risque pour construire une figure confiante de l'enfant autonome, s'il n'a pas le monopole sur les discours auxquels sont exposés les élèves, reste pourtant l'approche dominante au sein des séquences pédagogiques sur l'alimentation à l'école, et c'est à cette approche que souscrivent les entreprises de restauration dans leurs dispositifs.

\section{Dangers individuels, dangers collectifs}

Dans la vision ample que les dispositifs info-pédagogiques et communicationnels des entreprises de restauration collective proposent de l'alimentation, et malgré la réticence à expliciter leurs causes profondes, des menaces de différente nature sont associées aux aliments et aux pratiques alimentaires. Il s'agit de dangers qui peuvent toucher individuellement les plus jeunes à court ou moyen terme ou qui peuvent affecter à long terme 
la collectivité, voire la planète entière. Dans les dispositifs s'adressant à la fois aux élèves et à leurs parents les mauvais résultats scolaires sont présentés comme une des conséquences néfastes de " mauvaises " habitudes alimentaires : la peur de l'échec scolaire est évoquée dans le site de Sodexo, entreprise qui se donne pour mission de «favoriser le bien-être et la réussite des élèves » ainsi que dans la newsletter de Scolarest De bouche à oreille. $\mathrm{Ne}$ pas réussir la rentrée, ne pas arriver à faire face à une reprise "soutenue et souvent contraignante ", ne pas mobiliser toute la concentration et l'énergie pour pouvoir acquérir de nouveaux savoirs sont certains des dangers auxquels les enfants peuvent s'exposer à cause d'une diète déséquilibrée ou d'une consommation insuffisante de fruits et de légumes de saison. Des modes et types de consommation alimentaires qualifiés de " mauvais » sont considérés également comme la cause de résultats sportifs désastreux. Le même bulletin d'information indique des remèdes à ce propos : «Votre enfant sort de l'école et ce soir c'est judo ? Pas question d'être flagada sur le tatami [...] Des fruits secs, une orange, un yaourt, avec ça votre champion n'ira pas au tapis ».

Les principales conséquences négatives de conduites alimentaires « erronées» touchent le corps même des enfants. Avec le slogan " une bonne nutrition pour une bonne croissance », les kits pédagogiques de la Fondation Louis Bonduelle distribués lors de la « Journée du légume » dans les restaurants scolaires gérés par l'entreprise Elior suggèrent implicitement qu'une nutrition inadéquate puisse provoquer une croissance irrégulière. Une mauvaise nutrition et, en particulier, une diète déséquilibrée, pauvre en légumes sont présentées comme dommageables pour le développement de l'enfant et pour la santé de l'adulte à venir.

Dans les dispositifs info-pédagogiques et communicationnels des entreprises de restauration collective comme dans les documents pédagogiques ou de vulgarisation utilisés par, ou sélectionnés pour des enseignants et animateurs périscolaires (ouvrages documentaires, polycopiés utilisés en cours), la nature des risques encourus pour la santé reste peu développée, surtout en ce qui concerne les symptômes et la progression des maladies graves. La newsletter De bouche à oreille ne fait mention que de risques mineurs pour la santé des plus jeunes : "petits tracas de santé pour les enfants : rhumes à répétition, fatigue, peau asséchée ». Ailleurs, on présente à l'enfant ce qu'il a déjà éprouvé, ou ce qu'il connaît déjà (indigestion, diarrhées, caries, flatulence, fatigue, mauvaise performance sportive, virus) avec plus de détails que les maladies graves, évoquées par des références à un cœur malade, ou des artères qui se rétrécissent, la tristesse, mais sans renseignements sur la progressivité de ces maladies ni les risques cumulés ou dans le temps.

Les typologies fonctionnelles des aliments présentées aux enfants à l'école primaire offrent plutôt une image du « danger évité » que du danger encouru : les « aliments bâtisseurs » rendent les os solides, les « aliments énergétiques » 
permettent aux enfants de réfléchir, marcher et courir, les " aliments protecteurs » éloignent les microbes.

De plus, la causalité entre le comportement dangereux et les conséquences est souvent directe et immédiate (" tu grossis », le corps est « obligé » de stocker des graisses), avec téléscopage de plusieurs conséquences néfastes d'une mauvaise alimentation. Dans le livret Léo et la terre (2005) de l'INPES pour des enfants de primaire, qui véhicule les normes du PNNS, une « dame de cantine " dit à des enfants que s'ils mangent trop de frites ils vont à la fois grossir et être en mauvaise santé : « c'est gras et si vous en mangez trop vous serez en mauvaise santé et vous grossirez ». Le surpoids et l'obésité sont également présentés comme équivalents, comme le montre ce passage tiré d'un ouvrage publié chez Actes Sud Junior : "l'énergie de ces aliments riches est stockée sous forme de graisse. On parle d'obésité, qui augmente les maladies de cour, des artères, et rend la vie des gens bien triste " (Les Aliments à petits pas, 2008). Cette ellipse suggère qu'une prise de poids est sur un même plan que le problème de l'obésité. Si une vision plus nuancée de l'apparition des maladies cardio vasculaires apparaît parfois dans les ouvrages documentaires ciblant les élèves de l'école primaire, on note une simplification dans la représentation du déclenchement possible de la maladie pour accentuer le lien direct avec les habitudes alimentaires : «il existe des troubles qui sont aggravés par notre manière de manger... Une quantité trop importante de cholestérol dans notre sang, pouvant ainsi provoquer un rétrécissement de nos artères. Ceci peut favoriser la formation d'un caillot de sang dans nos artères et avoir des conséquences mortelles... Nous pouvons nous mettre à en fabriquer trop [de cholestérol] si nous mangeons beaucoup de graisses et buvons trop d'alcool " (Je sais ce que je mange, Magnard 2001). L'emploi du verbe " pouvoir » atténue la causalité, cependant le seul facteur de risque qui apparaît est l'alimentation. Ce raccourci renforce l'approche comportementaliste : l'enfant, construit en tant que responsable de sa santé, s'il est sous la menace de la maladie, en contrôle ainsi la clé. L'enfant peut être ainsi « rassuré » selon ce modèle positiviste qui lie un résultat à une cause unique et identifiable.

De plus, les documents scolaires ou de vulgarisation pour les enfants ont tendance à représenter le surpoids et l'obésité de façon humoristique, par le biais de dessins caricaturaux d'enfants très gros, qui semblent contredire le discours verbal plus sérieux. L'humour des illustrations construit un double message sur le surpoids et l'obésité, entre désordres nutritionnels et objets de dérision.

$\mathrm{Au}$-delà du comportement individuel, les (et surtout « nos ») modes de production et de consommation alimentaires collectifs sont mis en relation avec « crises sanitaires, pollution, impact sur la biodiversité ». Mais, exception faite pour l'huile de palme dont les effets nocifs sont présentés de façon détaillée dans le site de Scolarest, souvent les dangers pour l'environnement 
ne sont pas précisés ; on indique laconiquement qu'il faut protéger la «santé de la planète » et « réduire notre impact environnemental ». Les documents pédagogiques ou de vulgarisation d'orientation écologique, tels que ceux qui figurent dans le kit de la MRES « De mon assiette à ma planète » cherchent à sensibiliser le jeune enfant à propos des dangers pour la planète, l'agriculture intensive, les pesticides et le gaspillage. Cependant, le message est souvent vague, et reste sur un registre émotif plus qu'informatif. Dans Une seule Terre pour nourrir les hommes (Gallimard Jeunesse, 2009), par exemple, des images photographiques créent une vision esthétique de la nature et du travail des agriculteurs sur l'échelle locale et humaine ; ils offrent en revanche et par contraste, des photos de la terre dévastée par la déforestation et par l'agriculture intensive, sans explications : la volonté de susciter un engagement émotif chez le jeune lecture l'emporte sur une volonté d'apporter des informations détaillées et concrètes.

\section{Responsabilités individuelles, responsabilités collectives}

En s'appuyant sur les savoirs spécialisés et experts des nutritionnistes et des diététiciens et en s'insérant dans le sillage des lignes-guide du PNNS, les dispositifs info-pédagogiques et communicationnels produits par les entreprises de restauration collective insistent sur la nécessité d'adopter de «bonnes » pratiques alimentaires : «bien » manger, manger « mieux », manger " de tout " sont les conditions nécessaires pour ne pas mettre en danger le corps et les performances des enfants. Une alimentation saine, équilibrée et savoureuse est envisagée comme " un atout incomparable pour avoir toutes les cartes en main pour réussir sa scolarité » (Newsletter Scolarest). Ce que «bien manger » veut dire, les aliments à privilégier, les quantités et les temps des prises alimentaires sont au centre des dispositifs proposés aux élèves, envisagés comme des sujets vulnérables, mais aussi comme les responsables de leur futur, à sensibiliser et à impliquer par le biais de différentes activités ludo-pédagogiques.

Les entreprises de restauration scolaire se présentent comme des acteurs qui participent avec l' " ensemble de la communauté " composée des élèves, des parents, des enseignants et des institutions publiques à la réalisation du bien commun. En voulant mettre d'accord tout le monde et en encourageant tout le monde à coopérer dans l'intérêt de tous, les entreprises non seulement soulignent la dimension collective de l'action dans laquelle elles s'engagent, mais se proposent comme une sorte de médiateur capable de concilier les attentes des divers acteurs concernés sous l'égide des politiques publiques en matière d'alimentation. C'est, par exemple, le rôle affiché par Scolarest : «Bon pour le goût, bon pour la santé, bon pour la planète : découvrez le premier éco-restaurant scolaire qui a 3 fois bon ! "C'est Bon Pour Nous" accueille les enfants des écoles primaires et crée le lien entre équilibre alimentaire et équilibre de la planète. [...] Il a déjà séduit enfants, personnel enseignant 
et familles, et a été adopté par 250 restaurants scolaires ! La qualité et le caractère complet du concept lui ont permis de se voir attribuer le label PNA. Le Programme National pour l'Alimentation mobilise tous les acteurs de l'alimentation selon le principe que "Bien manger, c'est l'affaire de tous". »

Dans leurs dispositifs communicationnels, les entreprises de restauration scolaire essaient de valoriser leurs prestations à travers les différentes démarches mises en place pour lutter contre les risques associés à l'alimentation. Le choix des aliments et les initiatives contre le gaspillage sont par exemple mis en avant en tant que démarches « responsables » dont les bénéfices sont autant collectifs qu'individuels. Les entreprises de restauration collective se présentent également comme des acteurs engagés dans la promotion d'actions « responsables » et indiquent dans les dispositifs de communication adressés aux parents des lignes de conduite à suivre " pour revenir à une alimentation saine, sûre et authentique, sans dépenser plus » et pour protéger l'environnement dans l'intérêt de tous.

Les entreprises prestataires de services dans le secteur de la restauration scolaire s'attribuent surtout un rôle d'acteur engagé dans l'éducation alimentaire des élèves. «L'école mérite toute notre attention. [...] Éduquer au goût c'est aussi notre marque d'attention » affirme, par exemple, l'entreprise Elior. Par le biais d'animations, programmes d'information, kits infopédagogiques et initiatives diverses, les entreprises de restauration collective souhaitent s’insérer parmi les acteurs impliqués dans l'éducation alimentaire et visent à s'imposer comme des interlocuteurs fiables, compétents en matière de nutrition et disposant d'une connaissance approfondie des attentes, des préférences et des goûts des élèves : "Notre mission consiste aussi à venir en appui de toute la chaîne éducative en matière d'information nutritionnelle : chefs d'établissement, parents, enseignants...Quant à nos équipes, au-delà de leur savoir-faire culinaire, une formation est dispensée pour mieux comprendre les enfants et adolescents et adopter les meilleures attitudes pour atteindre la qualité de service attendue par nos clients ».

La réduction des problèmes de santé, tout comme la gestion des risques environnementaux liés à l'alimentation apparaissent, en effet, passer principalement par la transformation des comportements individuels des élèves. Les enfants et les adolescents sont interpellés directement, invités à rationnaliser leurs choix et habitudes alimentaires et à les conformer volontairement aux normes dictées par les savoirs des experts en nutrition et disséminées par les actions publiques. "Je fais attention à ma consommation d'eau ", " je privilégie les fruits et légumes de saison », " je respecte la biodiversité », " je trie mes déchets ${ }^{5}:$ dans ces discours responsabilisants, la multiplication des risques et des savoirs experts mobilisés pour la gestion de ces risques va de pair avec la multiplication des impératifs que les élèves sont censés

5 http://www.scolarest.fr/restauration/scolaire/geste-ecologique-responsable/agir-durable/29311 
appliquer à eux-mêmes et à leurs conduites. Parallèlement, la reconnaissance des compétences des enfants se traduit par un processus de responsabilisation qui pousse les plus jeunes à l'autocontrôle et à l'autosurveillance. Apprendre à gouverner ses propres comportements alimentaires devient un volet du processus d'acquisition des «techniques de soi » (Foucault, 1992).

De même, dans les documents sur la nutrition et l'alimentation produits pour les enseignants par le monde de l'édition et les acteurs du monde éducatif, l'enfant est souvent représenté comme seul dans sa démarche nutritionnelle. Ce curieux isolement de l'enfant ne prend pas en compte les différentes déterminations sociales et contextuelles qui influencent ses choix alimentaires et qui contribuent à former ses habitudes. Paradoxalement l'enfant est souvent montré en famille ou à l'école, justement dans des lieux ou des institutions où la construction de règles et de rites dépasse souvent l'individu. Le document INPES propose une affiche détachable montrant « 10 points pour rester en forme " à travers la voix de l'enfant qui dit « je prévois du temps de prendre un bon petit-déjeuner "; " je bouge, je prends l'habitude de me déplacer à pied », etc. L'évocation du rôle d'autres acteurs dans l'alimentation intervient non pas pour approfondir les causes mais pour aider le jeune enfant à mieux « se contrôler " : «si tu as du mal à te contrôler, parles-en à tes parents ou demande-leur de t'emmener voir un nutritionniste " (Les Aliments à petits pas). Le parent est partenaire de l'enfant qui doit aussi s'auto-diagnostiquer.

Alors que les textes officiels insistent sur une éducation à la santé comme étant l'affaire de tous (familles, équipes éducatives, partenaires) ${ }^{6}$, la mise en œuvre d'une éducation à la santé met surtout l'accent sur la responsabilisation de l'élève individuel et « vise à aider chaque jeune à s'approprier progressivement les moyens d'opérer des choix, d'adopter des comportements responsables ${ }^{7}$ ». La volonté de construire le personnage de l'enfant comme seul responsable de ses actes explique la réticence, comme nous l'avons vu, à insister sur les racines familiales, psychologiques ou héréditaires d'un problème nutritionnel qui renverraient à la vie familiale, à un cas particulier médical ou aux valeurs culturelles. Cette autonomie de l'enfant permet notamment de créer un espace éducatif débarrassé des déterminants socio-économiques ou génétiques, pour dresser le portrait de l'enfant maître de son destin et ainsi faciliter la transmission d'un message de responsabilisation, voire de culpabilisation de l'individu.

L'approche écologique de certains des ouvrages de vulgarisation amène les auteurs et éditeurs à construire l'enfant non seulement comme maître de ses choix alimentaires, mais aussi comme consommateur actif et engagé, qui adopte des gestes individuels de tri, qui apprend à lire des étiquettes sur des produits alimentaires pour être bien informé, qui évite les sacs en plastique

6 Bulletin Officiel circulaire du 25 avril 2002, « Politique de santé en faveur des élèves ».

7 « Orientations pour l'éducation à la santé à l'école et au collège », BOEN, nº 45 du 3 décembre 1998. 
et les suremballages ${ }^{8}$. Cette "rhétorique de la responsabilité individuelle » normalisante et universalisante, largement déployée dans les documents de sensibilisation aux problèmes environnementaux, a tendance à déléguer à l'individu (plutôt qu'à l'industrie) la mise en place des solutions écologiques (Comby, 2013).

Les ouvrages documentaires pour jeunes conçus selon cette orientation écologique et solidaire, mettent l'accent sur les valeurs de partage et de communauté et ainsi inscrivent ces gestes individuels dans une vision idéale de culture culinaire, gastronomique. Dans Une seule Terre pour nourrir les hommes (Gallimard Jeunesse 2009), préfacé par Pierre Rabhi fondateur du mouvement Colibris pour la terre, c'est la tradition française du repas partagé qui constitue une barrière contre les maux nutritionnels : "Le "paradoxe français" est qu'au pays des mille fromages, du pain et du vin, on souffre moins d'obésité et de maladies cardiaques qu'ailleurs : cela tient à la régularité des repas et à une vraie cuisine familiale ». Si les pratiques culturelles collectives sont représentées comme remède aux problèmes de nutrition et d'agriculture intensive et industrielle, la vie associative et l'action politique sont aussi mises en avant pour montrer les niveaux d'intervention possibles, en partant de l'individu pour s'ouvrir à " mes parents ", " ma ville » " ma région " « l'Union européenne » et " la communauté internationale ». Ces principes de citoyenneté et de participation, assez exceptionnels dans les documents didactiques sur l'alimentation, rejoignent pourtant l'esprit même et les ambitions des différentes " éducation à » (éducation au développement durable, éducation à la santé, éducation à la citoyenneté) qui font leur entrée au sein de l'école, encourageant les équipes pédagogiques à mettre en place des projets interdisciplinaires à l'échelle de l'établissement. On peut dire que la promotion de la solidarité et de l'action collective par l'entreprise vient combler une des lacunes du système éducatif français qui a du mal à intégrer un esprit d'action collectif dans le cadre de l'enseignement traditionnel (Kovacs, 2011).

\section{Conclusion}

La scénarisation du risque dans les dispositifs info-pédagogiques et communicationnels sur l'alimentation conçue par les entreprises de restauration collective ressemble donc à plusieurs égards à celle qu'on peut observer dans les documents sur la nutrition produits par les acteurs institutionnels travaillant en partenariat avec l'école (INPES, maisons d'édition proches du monde scolaire) ou sélectionnés par des médiateurs (documentalistes, organismes comme le CNDP) pour répondre aux préoccupations du corps enseignant. Un discours fonctionnaliste sur la nutrition, qui définit le risque comme la conséquence mal définie d'un comportement alimentaire à

8 Voir par exemple l'ouvrage L'alimentation publié chez Milan Jeunesse en 2005, collection « Agir pour ma planète ». 
proscrire, sert à la fois les ambitions circonscrites des programmes d'éducation nutritionnelle à l'école, et conforte le rôle de l'école républicaine " pour tous " en faisant abstraction d'un ensemble de facteurs de risque émanant des contextes socio-économiques ou psychologiques des familles. Ce discours permet à l'entreprise de se montrer solidaire d'une philosophie éducative de l'autonomie qui responsabilise les jeunes élèves en offrant un message positif, et positiviste, de leur capacité d'auto-gouvernance. Ce discours de l' « empowerment " rend l'entreprise partenaire potentiel des initiatives qui font la promotion d'un agir collectif, mais surtout, et paradoxalement, d'une attitude individuelle et d'une capacité à la gestion individuelle du risque. En épousant la rhétorique d'une rationalisation des comportements alimentaires, l'entreprise participe à la légitimation et à la circulation, à l'école, des savoirs et des pratiques sur l'alimentation qui restent décontextualisés car associés à des normes réductrices qui ne traduisent pas la complexité de l'alimentation en tant que fait social.

$\mathrm{La}$ construction et la diffusion des risques liés à l'alimentation s'accompagnent de changements qui touchent l'ensemble des acteurs concernés. Pour les entreprises de restauration collective la prise en compte des risques alimentaires se traduit non seulement par une réorganisation de leurs activités sur le plan gestionnaire, mais aussi par une redéfinition des stratégies marketing, un élargissement de l'offre de services et l'acquisition de nouveaux savoirs et savoir-faire. Au sein des entreprises de restauration collective, de nouvelles compétences communicationnelles et pédagogiques sont actuellement en train de se développer en lien avec l'endossement par ces organisations d'un nouveau rôle social et éducatif. En se présentant comme des acteurs engagés dans le développement durable et notamment dans la lutte contre l'obésité et les déséquilibres alimentaires, les entreprises de restauration collective essayent de se doter de nouvelles connaissances et techniques afin de développer des programmes d'information nutritionnelle et d'éducation environnementale pour les élèves de la maternelle au lycée. Parallèlement dans les écoles, les équipes pédagogiques sont amenées à revoir une approche disciplinaire traditionnellement portée sur le "savoir " afin de prendre en compte les préconisations ministérielles pour une éducation à la santé et à la nutrition qui formerait, à partir de nouveaux «savoir-faire » des sujets et des citoyens capables de prendre des décisions raisonnées. Chez les enseignants et directeurs d'établissement, ce changement provoque parfois un sentiment de gêne, voire de refus : ils sont au courant que l'éducation nutritionnelle a pour effet de "s'immiscer » dans le domaine familial'. L'enseignant, s'il semble privilégier les dispositifs sur l'alimentation qui n'abordent que très peu les risques et les dangers, s'aligne à un discours qui lui semble le plus neutre

9 Nous avons recueilli l'avis de deux directeurs d'école primaire et de quatre enseignants, lors d'entretiens sur leurs pratiques dans les enseignements sur l'alimentation. 
possible face à ce nouveau défi qui modifie les attentes sociales par rapport à son rôle dans la vie de l'enfant.

\section{BIBLIOGRAPHIE}

BECK Ulrich, La société du risque. Sur la voie d'une autre modernité, Paris, Aubier, 2001.

COMBY Jean-Baptiste, "Faire du bruit sans faire de vagues ", Communication [En ligne], Vol. 31/2 | 2013, mis en ligne le 18 septembre 2013, consulté le 02 février 2014. URL : http://communication.revues.org/4439 ; DOI : 10.4000/communication.4439

DE IULIO Simona, «De la peur de manquer aux dangers de l'obésité : les discours publicitaires et la construction sociale des risques alimentaires ", Le temps des médias, $\mathrm{n}^{\circ} 17,2011$, p. 143-163.

DUBUISSON-QUELLIER Sophie, " Le prestataire, le client et le consommateur. Sociologie d'une relation marchande ", Revue française de sociologie, vol. 40, n 4, 1999, p. 671-688.

FOUCAULT Michel, "Technologies of the self ", dans Martin, L., Gutman, H., Hutton, P. (sous la dir. de), Technologies of the Self: A Seminar with Michel Foucault, London, Tavistock, 1988.

GIDDENS Anthony, Les conséquences de la modernité, Paris, L'Harmattan, 1994.

HELLER René, Jacquot Raymond, Moyse Alexis, Pascaud Marc, " Nutrition ", Encyclopaedia Universalis [en ligne], http://www.universalis.fr/encyclopedie/nutrition/ [consulté le 31 janvier 2014].

HILGARTNER Stephen, «The social construction of risk objects. Or, how to pry open networks of risk » dans Short James F. et Clarke Lee, (sous la dir. de) Organizations, Uncertainties and Risk, Boulder, Westview Press, 1992, p. 39-53.

JEANNERET Yves, Penser la trivialité. Volume 1, La vie triviale des êtres culturels, Paris, Lavoisier \& Hermes-sciences, 2008.

JOURDAN Didier et al., «Éducation à la santé à l'école : pratiques et représentations des enseignants du primaire », Santé Publique, vol. 14, n 4, 2002, p. 403-423.

KOVACS Susan, «Projets environnementaux et communication “verte” à l'école : étude comparative de quatre collèges dans le Nord et le Bas Rhin » Recherches en communication, $\mathrm{n}^{\circ}$ 35, 2011, p. 155-170.

LAGADEC Patrick, La civilisation du risque. Catastrophes technologiques et responsabilité sociale, Paris, Seuil, 1981.

LUPTON Deborah, Risk, London, Routledge, 1999.

MANDERSCHEID Jean-Claude, PITHON Gérard, « Eléments de réflexion pour une pédagogie de l'éducation à la santé en milieu scolaire ", Spirale - Revue de Recherches en Éducation, n 25, 2000, p. 49-68.

Résumé : Cet article propose une analyse comparative de la rhétorique des risques alimentaires présente dans les dispositifs d'information et de communication à destination des élèves de l'école primaire, produits d'un côté par les organisations qui travaillent 
dans le secteur de la restauration scolaire, et de l'autre, par les acteurs - éditeurs et enseignants - proches du monde scolaire. Les stratégies discursives mises en place par ces deux groupes d'acteurs pour construire la notion de risque relèvent d'un discours normé, à la fois culpabilisant et rassurant, où les dangers apparaissent à la fois réels et insaisissables. Un discours fonctionnaliste sur la nutrition sert à la fois les pratiques des enseignants parfois réservés face aux ambitions des programmes d'éducation nutritionnelle à l'école et permet à l'entreprise de se montrer solidaire d'une philosophie éducative de l'autonomie qui responsabilise les jeunes élèves en offrant un message positif, et positiviste, de leur capacité d'auto-gouvernance.

Mots-clés : risque alimentaire, dispositif info-pédagogique, support d'enseignement, entreprise de restauration, école primaire, éducation nutritionnelle, édition pour la jeunesse, vulgarisation scientifique.

Abstract: This article offers a comparative analysis of the rhetoric of risk found in pedagogical devices produced for school children by food service companies and by editors and teachers. Discursive strategies used to construct the idea of risk are highly normalized, both responsibilizing and reassuring, and portray food risks as both real and undefined. A functionalist and positivist approach to nutrition, which emphasizes childrens' autonomy and capacity for self-governance, serves the needs of both teachers, faced with difficult choices due to the emerging importance of health education in school curricula, and the new image of the corporate actor as educator.

Keywords: food risk, pedagogical device, pedagogical document, food service company, primary school, nutrition education, childrens' books, popular science. 